\title{
RE: CA 19-9 in Potentially Resectable Pancreatic Cancer: Perspective to Adjust Surgical and Preoperative Therapy
}

\author{
Richard J. Bold, MD \\ Department of Surgery, University of California, Davis Medical Center, Sacramento, CA
}

I am really not a pessimist, but several concepts continue to surround the surgical management of pancreatic adenocarcinoma: (1) despite improvements in radiologic technology, a significant fraction of patients are still found to have unresectable disease in the operating room, (2) despite improvements in perioperative care and team-based patient management, a significant fraction of patients develop complications of surgical resection that may lead to immediate death, delayed death, or dramatically decreased quality of life, (3) the vast majority of patients will die from recurrent disease despite a ãcurativeã resection, and (4) improvements in adjuvant therapy have lagged behind the treatment of other solid organ malignancies and offer only small improvements in median survival. If I were a pessimist, I would never operate on these patients, but instead I continue to hold out hope that each patient may be that uncommon 5- or 10-year survivor following surgical resection of pancreatic cancer.

Nearly a decade has passed since the association of carbohydrate antigen (CA) 19-9 with some aspect of the biology of pancreatic cancer was first reported. Since that time, various roles of CA 19-9 in the management of patients with pancreatic adenocarcinoma have been described including the differentiation of benign from malignant pancreatic disease, prediction of resectable disease, identification of patients for selective staging laparoscopy, the identification of patients at risk for early recurrence and death following potentially curative resection, and for use in the surveillance for disease recurrence. Yet for each of these aspects, the specific role of CA 19-9 is only part of clinical assessment and decision making in these patients. Before beginning the discussion, it is critical

(C) Society of Surgical Oncology 2013

Published Online: 7 April 2013

R. J. Bold, MD

e-mail: richard.bold@ucdmc.ucdavis.edu to understand what CA 19-9 is and how it can be used as a ã tumor marker.ã CA 19-9 was first identified following the screening patient sera by hybridoma-derived monoclonal antibodies. One such antibody, 1116 NS 19-9 developed by Koprowski et al., ${ }^{1 \tilde{3} 3}$ was shown to react with a sialylated lacto- $N$-fucopentaose II, which was present in high levels in sera of patients with colon, gastric, and pancreatic cancer. Based on the screening of a variety of tumors by immunohistochemical methods as well as sera from patients with a variety of gastrointestinal and gynecologic cancers, CA 19-9 was felt to be specific for malignancy and termed a ã tumor markerã ${ }^{4}$ In screening sera from more than 1,000 blood donors, CA 19-9 was found to be undetectable inô $8 \hat{o} \%$, and wasô $<37 \mathrm{U} / \mathrm{ml}$ in all but six samples, hence the threshold value of $37 \mathrm{U} / \mathrm{ml}$ for ã normalã ..$^{5}$ Yet $4 \hat{o} \%$ of a parallel study population of patients with benign disease (such as inflammatory bowel disease, pancreatitis, or gastrointestinal polyps) had CA 19-9 levelsô $>37 \mathrm{U} / \mathrm{ml}^{5}{ }^{5}$ Thus, CA $19-9$ is not a perfect tumor marker in terms of sensitivity (79ô\% for pancreatic adenocarcinoma, 50ô\% for gastric carcinoma, and $46 \hat{0} \%$ for advanced colorectal carcinoma) and specificity (98.5ô\%). However, it is pretty good when compared with other tumor markers such as CEA, alphafetoprotein or CA $125 .^{6,7}$

There are three important limitations of CA 19-9 that must be recognized as they are critical to the implementation in patients with suspected pancreatic cancer. The first is that CA 19-9 is a sialylated Lewis (a) antigen, which is normally expressed on the surface of erythrocytes. Up to $10 \hat{\%} \%$ of the white population will not express any detectable level of CA 19-9 because of absence of fucosyltransferase, one of the enzymes involved in the biosynthesis. ${ }^{8}$ Therefore, in up to $10 \hat{0} \%$ of patients with pancreatic adenocarcinoma, CA 19-9 will be undetectable and therefore of no utility in any aspect of clinical decision making. The second aspect relates to the lack of perfect specificity in which patients with benign disease can have 
modest elevations of CA 19-9. The most common situation in which this impacts surgical decision making is in the patient without a radiologically identified mass or without histopathologic evidence of a pancreatic malignancy. Fortunately, this is infrequent, and the patient is usually relieved to hear of a benign process when a pancreaticoduodenectomy has been performed based on clinical suspicion in these circumstances. The last aspect may be more problematic for the surgeon as CA 19-9 is excreted by the liver into the biliary system; therefore, obstructions of the biliary system may cause elevations that do not accurately reflect either the underlying process or the extent of malignancy. This is not simply due to the underlying process causing elevation, as biliary decompression results in dramatic reduction of serum CA 19-9 levels. ${ }^{9,10}$ Several authors have suggested that ã correctingã for the degree of biliary obstruction by normalizing the CA 19-9 by the serum bilirubin improves the differentiation of benign from malignant disease. ${ }^{11,12} \mathrm{We}$ and others have also suggested that this correction may slightly improve the relationship between CA 19-9 level and extent of disease in pancreatic cancer. ${ }^{13 a ̃} 15$

In the current issue of Annals of Surgical Oncology, Hartwig et al. ${ }^{16}$ add to the literature with one of the largest series of patients (more than 1,500) that again demonstrates that elevated CA 19-9 is associated with advanced/unresectable pancreatic adenocarcinoma despite preoperative radiologic studies demonstrating resectability. Furthermore, the authors report that preoperative CA 19-9 levels are prognostic following resection and finally that the persistence of elevation postoperatively portends an extremely short survival. From these analyses, the authors propose an algorithm to be used in guiding the therapy of patients with presumed resectable disease. Each aspect of this analysis has been reported previously, albeit in smaller series or as individual components on the relationship of CA 19-9 to the biology of pancreatic adenocarcinoma. For example, the authors note that in a cohort of 533 patients with chronic pancreatitis for whom CA 19-9 levels were available, the accuracy of an elevated CA 19-9 was 76.5ô $\%$. As noted previously, there is a significant overlap of CA 19-9 values between patients with chronic pancreatitis and pancreatic adenocarcinoma (both elevated in benign conditions, and normal in malignant disease) such that clinicians have been discouraged from using CA 19-9 in the diagnostic differentiation of these two entities. ${ }^{17}$

The algorithm proposed by the authors includes two decision points in which the absolute value of CA 19-9 is used to guide therapeutic intervention. Although the threshold for choosing one treatment plan versus another remains controversial, discussion about how CA 19-9 is used to guide these decisions is warranted. The first decision point is following radiologic evaluation of pancreatic adenocarcinoma with findings demonstrating a resectable tumor. The authors propose that if the CA 19-9 is ã highly elevatedã (and they propose a threshold of 1,000 U/ml), then laparoscopy should be performed and neoadjuvant therapy should be administered. The first recommendation is well supported by other studies, and most pancreatic surgeons have adopted selective laparoscopy based on elevations of CA 19-9 as well as other criteria such as pancreatic tail tumors or locally advanced disease on radiologic studies. However, neoadjuvant therapy has not become a standard practice despite a variety of theoretical benefits. ${ }^{18}$ The primary issue with this approach is that there is nothing to suggest that patients with very high levels or CA 19-9 are more likely to benefit from neoadjuvant therapy; simply put, CA 19-9 may be an important prognostic factor, but it fails as a predictive marker. Although some authors suggest that patients with highly elevated preoperative CA 19-9 have poor survival and perhaps should be screened more carefully for the assessment of resectable disease, there is no available molecular, histologic, or biochemical marker to guide patient selection for neoadjuvant therapy. Therefore, this approach should be an all-or-none regimen; namely all patients are considered or all patients proceed directly to surgical therapy.

The last decision point is following surgical resection and the determination of whether CA 19-9 is increasing or decreasing. In the setting of an increasing postoperative CA 19-9, the authors propose the delivery of combination adjuvant therapy or the omission of adjuvant therapy. This is based on the observation that these patients have a high risk of early disease recurrence and poor prognosis. This recommendation is based on two assumptions: (1) ã combination adjuvant therapyã (the authors denote PEFG or gemcitabine/erlotinib) has improved outcome compared to standard adjuvant therapy, and (2) some patient do so poorly that adjuvant therapy has no benefit. Unfortunately, the survival benefit of combination therapies over singleagent gemcitabine is modest; furthermore, most oncologists would recommend the best adjuvant therapy to all suitable patients. In addition, other than performance score, there is limited information to identify which patients will not receive any benefit from postoperative adjuvant and therefore withholding potentially life-prolonging therapy from suitable patients does not seem reasonable. Therefore, while persistent elevations of CA 19-9 in the postoperative setting are prognostic and may be used to counsel patients about anticipated outcomes, the predictive role in guiding therapy has not been validated and should be discouraged, especially if considering withholding therapy.

In summary, Hartwig et al. have added to the burgeoning information surrounding CA 19-9 in pancreatic adenocarcinoma and its use in the preoperative and postoperative management of patients. Unfortunately, there 
remain limitations in its applicability to surgical patients and controversy as to the appropriate threshold for decision making, particularly for the identification of unresectable patients. Clearly, additional predictive markers of outcome to all aspects of therapy in pancreatic cancer are needed, but until then, CA 19-9 will remain the standard. ${ }^{19}$

\section{REFERENCES}

1. Koprowski H, Steplewski Z, Mitchell K, Herlyn M, Herlyn D, Fuhrer P. Colorectal carcinoma antigens detected by hybridoma antibodies. Somat Cell Genet. 1979;5:957ã 71.

2. Koprowski H, Herlyn M, Steplewski Z, Sears HF. Specific antigen in serum of patients with colon carcinoma. Science. 1981;212:53ã 5 .

3. Magnani JL, Nilsson B, Brockhaus M, Zopf D, Steplewski Z, Koprowski H, Ginsburg V. A monoclonal antibody-defined antigen associated with gastrointestinal cancer is a ganglioside containing sialylated lacto- $N$-fucopentaose II. J Biol Chem. 1982;257:14365ã 9 .

4. Del Villano BC, Zurawski VR Jr. The carbohydrate antigenic determinant 19-9 (CA 19-9): a monoclonal antibody defined tumor marker. Lab Res Methods Biol Med. 1983;8:269ã 82.

5. Del Villano BC, Brennan S, Brock P, Bucher C, Liu V, McClure $\mathrm{M}$, et al. Radioimmunometric assay for a monoclonal antibodydefined tumor marker, CA 19-9. Clin Chem. 1983;29:549ã 52.

6. Bates SE, Longo DL. Tumor markers: value and limitations in the management of cancer patients. Cancer Treat Rev. 1985;12: 163 ã 207.

7. Jacobs EL, Haskell CM. Clinical use of tumor markers in oncology. Curr Probl Cancer. 1991;15:299ã 360.

8. Miyoshi E, Moriwaki K, Nakagawa T. Biologic function of fucsylation in cancer biology. J Biochem. 2008;143:726ã 9 .

9. Korkmaz M, Unal H, Selcuk H, Yilmaz U. Extraordinarily elevated serum levels of CA 19-9 and rapid decrease after successful therapy: a case report and review of the literature. Turk J Gastroenterol. 2010;21:461ã 3 .

10. Peterli R, Meyer-Wyss B, Herzog U, Tondelli P. CA 19-9 has no value as a tumor marker in obstructive jaundice. Schweiz Med Wochenschr. 1999;23:77ã 9.

11. Mann DV, Edwards R, Ho S, Lau WY, Glazer G. Elevated tumor marker CA 1-9: clinical interpretation and influence of obstructive jaundice. Eur J Surg Oncol. 2000;26:474ã 9.

12. La Greca G, Sofia M, Lombardo R, Latteri S, Ricotta A, Puleo S, et al. Adjusting CA19-9 values to predict malignancy in obstructive jaundice: influence of bilirubin and C-reactive protein. World J Gastroenterol. 2012;18:4150ã 5.

13. Schlieman MG, Ho HS, Bold RJ. Utility of tumor markers in determining resectability of pancreatic cancer. Arch Surg. 2003; 138:951ã 5.

14. Ong SL, Sachdeva A, Garcea G, Gravante G, Metcalfe MS, Lloyd DM, et al. Elevation of carbohydrate antigen 19.9 in benign hepatobiliary conditions and its correlation with serum bilirubin concentration. Dig Dis Sci. 2008;53:3213ã 7.

15. Kang CM, Kim JY, Choi GH, Kim KS, Choi JS, Lee WJ, et al. The use of adjusted preoperative CA 19-9 to predict the recurrence of resectable pancreatic cancer. J Surg Res. 2007;140:31ã 5.

16. Hartwig A, Strobel O, Hinz U, Fritz S, Hackert T, Roth C, et al. CA 19-9 in potentially resectable pancreatic cancer: perspective to adjust surgical and preoperative therapy. Ann Surg Oncol. 2012. doi:10.1245/s10434-012-2809-1. (Epub ahead of print).

17. Locker GY, Hamilton S, Harris J, Jessup JM, Kemeny N, et al. ASCO 2006 update of recommendations for the use of tumor markers in gastrointestinal cancer. J Clin Oncol. 2006;24: $5313 a ̃ 27$.

18. Crane CH, Varadhachary G, Wolff RA, Pisters PW, Evans DB. The argument for pre-operative chemoradiation for localized, radiographically resectable pancreatic cancer. Best Pract Res Clin Gastroenterol. 2006;20:365ã 82.

19. Brody JR, Witkiewicz AK, Yeo CJ. The past, present, and future of biomarkers: a need for molecular beacons for the clinical management of pancreatic cancer. Adv Surg. 2011;45:301ã 21 . 